\title{
SOLUTIONS FOR AN INFINITE COMPRESSIBLE NONLINEARLY ELASTIC BODY UNDER A LINE LOAD
}

\author{
$\mathrm{BY}$ \\ PAUL G. WARNE (Department of Mathematics, The Webb School of Knoxville, Knoxville) \\ AND \\ DEBRA A. POLIGNONE (Department of Mathematics, University of Tennessee, Knoxville)
}

\begin{abstract}
The axisymmetric deformation of a nonlinearly elastic isotropic compressible infinite elastic body subjected to a concentrated vertical line load is considered. We first derive the solution to this problem within the context of the linear theory of elasticity. We then obtain the governing equations for the nonlinear problem via the Principle of Stationary Potential Energy, and use these equations to obtain classes of compressible finite elasticity solutions for the line load problem. Finally, a comparison with finite anti-plane shear of compressible isotropic materials is made.
\end{abstract}

1. Introduction. Elasticity problems involving a concentrated load acting on an infinite or semi-infinite body have been considered for over a century. The linearly elastic Boussinesq problem is to find a solution of the linearized equations of elasticity for an isotropic half space under a point load perpendicular to the boundary. Boussinesq's solution of 1885 for the radial and vertical displacements in cylindrical coordinates may be found in [1, pp. 398-402] (see also [2, p. 348; 3, p. 341]). The Mindlin problem in the theory of linear elasticity is to find the deformation due to a concentrated force applied at an arbitrary point of the semi-infinite body. Thus, the linear Boussinesq problem is a special case of the Mindlin problem. The linear displacements of Mindlin's problem may be found in [4, pp. 347-348]. The Kelvin problem is to determine the deformation of the half-space due to a concentrated vertical line load through the body, and can thus be determined by integrating Mindlin's solutions along the line load (see [5, pp. 16C162]). While all of these solutions are free-space Green's functions for the axisymmetric equations of linear elasticity (which, as is well known, may be reduced to the biharmonic equation), they suffer two deficiencies: the displacement under the load is infinite in each problem, thereby violating the basic premise of linearized elasticity, and some of the particles in the body lying on the line of action of the load pass through one another.

To remedy these fundamental physical defects, study of the nonlinearly elastic versions of such problems is warranted. Simmonds and Warne [6] have recently investigated the Boussinesq problem for both compressible and incompressible isotropic materials using

Received December 6, 1993 and in revised form March 29, 1994.

1991 Mathematics Subject Classification. Primary 73G05, 73C02, 73C50, 73B99.

(c) 1996 Brown University 
the exact equations of nonlinear hyperelasticity, while the problem of a concentrated vertical line load applied to a nonlinearly elastic rubber-like plate was considered by Simmonds [7] and Simmonds and Horn [8]. In this paper, we examine the problem of a nonlinearly elastic infinite compressible body subjected to a concentrated vertical line load, and thus avoid certain boundary conditions which complicate but in general do not add to the understanding of the deformation in the vicinity of the load. In addition to their intrinsic interest and connection with the plate problem, solutions for this problem can be used to solve the nonlinear Kelvin problem (see [5, pp. 14-17]). In Sec. 2, we formulate our problem in a variational framework using the Principle of Stationary Potential Energy. In Sec. 3, we derive the solution to the linearized version of our problem. In Sec. 4, we obtain the Euler differential equations associated with the nonlinear variational functional, and use these to determine classes of nonlinearly elastic compressible materials which admit the linear solution as a solution to the fully nonlinear problem. Finally, in this section, we draw an interesting comparison between the problem studied here and the anti-plane shear problem studied by Polignone and Horgan [9].

2. Problem formulation. Relative to a fixed right-handed Cartesian reference frame $O x y z$, the axisymmetric deformation of an isotropic compressible infinite elastic space under a constant line load of magnitude $\hat{\alpha}$ per unit length along the $z$-axis is considered. Let $\left\{\mathbf{e}_{x}, \mathbf{e}_{y}, \mathbf{e}_{z}\right\}$ denote the orthonormal base vectors associated with $O x y z$ and let $(r, \theta, z)$ denote a set of circular cylindrical coordinates in this frame with associated orthonormal base vectors $\left\{\mathbf{e}_{r}, \mathbf{e}_{\theta}, \mathbf{e}_{z}\right\}$, where

$$
\mathbf{e}_{r}=\mathbf{e}_{x} \cos \theta+\mathbf{e}_{y} \sin \theta, \quad \mathbf{e}_{\theta}=-\mathbf{e}_{x} \sin \theta+\mathbf{e}_{y} \cos \theta
$$

Also, let

$$
\mathbf{x}=r \mathbf{e}_{r}(\theta)+z \mathbf{e}_{z}, \quad 0 \leq r<\infty \text { and } \quad-\infty<z<\infty,
$$

denote the position of a particle in the reference shape of the body. We assume that, when subjected to a body force per unit volume, $\mathbf{p}=-p_{z}(r, z) \mathbf{e}_{z}$, the body deforms axisymmetrically. Thus, the deformed position of the particle has the form

$$
\overline{\mathbf{x}}=\bar{r}(r) \mathbf{e}_{r}(\theta)+[z+v(r)] \mathbf{e}_{z}
$$

where $\bar{r}(r)$ and $v(r)$ are unknown functions assumed to be sufficiently smooth for all derivatives in what follows to exist. Since the deformation in every plane perpendicular to the $z$-axis is the same it is sufficient to consider any two such planes a unit depth apart. Within these planes we take

$$
p_{z}(r, z)=p_{z}(r)=\frac{\hat{\alpha} \delta(r)}{2 \pi r}
$$

where $\hat{\alpha}$ has the dimensions of FORCE/LENGTH, and $\delta(\bullet)$ is the Dirac delta function[al].

The deformation gradient $\mathbf{F}$ is defined by $d \overline{\mathbf{x}}=\mathbf{F} \bullet d \mathbf{x}$ and the right Cauchy-Green deformation tensor $\mathbf{G}=\mathbf{F}^{\mathrm{T}} \bullet \mathbf{F}$; thus, $d \overline{\mathbf{x}} \bullet d \overline{\mathbf{x}}=d \mathbf{x} \bullet \mathbf{G} \bullet d \mathbf{x}$. The physical components 
of $\mathbf{G}$ in circular cylindrical coordinates follow from this last expression and (2.3) as

$$
\mathbf{G}=\left[\begin{array}{ccc}
\bar{r}^{\prime 2}+v^{\prime 2} & 0 & v^{\prime} \\
0 & \bar{r}^{2} / r^{2} & 0 \\
v^{\prime} & 0 & 1
\end{array}\right]
$$

with corresponding standard strain invariants ([10, p. 57])

$$
\begin{aligned}
& I_{1}=\operatorname{tr} \mathbf{G}=\bar{r}^{\prime 2}+v^{\prime 2}+1+\frac{\bar{r}^{2}}{r^{2}}, \\
& I_{2}=\frac{1}{2}\left[(\operatorname{tr} \mathbf{G})^{2}-\operatorname{tr} \mathbf{G}^{2}\right]=\frac{\bar{r}^{2}}{r^{2}}\left(\bar{r}^{\prime 2}+v^{\prime 2}+1\right)+\bar{r}^{\prime 2}, \\
& I_{3}=\operatorname{det} \mathbf{G}=\frac{\bar{r}^{2}}{r^{2}} \bar{r}^{\prime 2} .
\end{aligned}
$$

Here and elsewhere, $\left({ }^{\prime}\right)$ will always denote the derivative of a function with respect to its argument.

The strain energy per unit undeformed volume of a homogeneous isotropic nonlinearly elastic material may be written in the form

$$
W=\mu w\left(I_{1}, I_{2}, I_{3}\right)
$$

where $\mu$ is an elastic modulus and $w$ is a dimensionless strain energy density. Recalling the statement prior to Eq. (2.4), the load potential, which follows from the principle of virtual work, is of the form

$$
\Psi=-2 \pi \int_{0}^{1} \int_{0}^{\infty} p_{z}(r)[z+v(r)] r d r d z
$$

where $p_{z}(r)$ is found in (2.4). The potential energy, $\Xi$, may then be expressed as

$$
\Xi[\bar{r}, v]=2 \pi \mu \int_{0}^{1} \int_{0}^{\infty} w r d r d z+\Psi .
$$

Since it is convenient in what follows to nondimensionalize, we let

$$
\kappa=\left(\frac{\hat{\alpha}}{2 \pi \mu}\right)^{1 / 3}, \quad(r, z)=\kappa(\rho, \zeta), \quad(\bar{r}, v)=\kappa(\bar{\rho}, \omega), \quad \Xi=\frac{\kappa}{\hat{\alpha}} \Pi
$$

to obtain the scaled functional

$$
\begin{aligned}
\Pi & \equiv \frac{\kappa}{\hat{\alpha}} \Xi[\bar{\rho}, \omega] \\
& =\kappa\left[\int_{0}^{1 / \kappa} \int_{0}^{\infty} w \rho d \rho d \zeta+\int_{0}^{1 / \kappa} \int_{0}^{\infty} \delta(\rho)(\zeta+\omega(\rho)) \rho d \rho d \zeta\right]
\end{aligned}
$$

Since $w$ is independent of $\zeta$, and $\delta(\bullet)$ is the Dirac delta function, (2.13) reduces to

$$
\Pi=\int_{0}^{\infty} w \rho d \rho+\omega(0)+\frac{1}{2 \kappa},
$$


and $\left(\bar{\rho}^{*}, \omega^{*}\right)$ is a stationary value of the variation of $(2.14)$ if and only if it is a stationary value of the variation of the functional

$$
\Pi[\bar{\rho}, \omega]=\int_{0}^{\infty} w \rho d \rho+\omega(0) .
$$

We require the strain energy $w$ to be continuous and piecewise smooth and that $\Pi$ be finite, and we seek solutions $\bar{\rho}^{*}(\rho)$ and $\omega^{*}(\rho)$ that render $\Pi$ stationary $(\delta \Pi=0)$. To guarantee the existence of a solution, constitutive requirements must be imposed on the strain energy $w$. Existence is guaranteed under the standard mathematical hypothesis that $w$ is convex with respect to the gradients of the deformed coordinates, in conjunction with reasonable smoothness and growth assumptions [11, p. 338]. But convexity of $w$ is unacceptable physically since it conflicts with the assumption that $w$ is frame invariant $[11$, p. 338]. Thus, subtler constitutive requirements are needed for $w$. Conditions that guarantee existence and allow a physically reasonable response for problems similar to the ones defined above are given by Ball in [11, pp. 375, 376]. Most of the strain energy densities proposed in the literature satisfy these conditions [11, pp. 388-392].

3. The linear solution. We wish now to derive the fields $\left(\bar{\rho}^{*}(\rho), \omega^{*}(\rho)\right)$ that render the dimensionless quadratic functional for the linearized line load problem stationary. The governing equations for the linear problem can be reduced to the axisymmetric biharmonic equation for the Love stress function $\phi$,

$$
\nabla^{2} \nabla^{2} \phi=0, \quad \nabla^{2}=\frac{\partial^{2}}{\partial r^{2}}+\frac{1}{r} \frac{\partial}{\partial r}+\frac{\partial^{2}}{\partial z^{2}}
$$

Since the displacements are independent of $z,(3.1)$ reduces to the ordinary differential equation

$$
\phi^{\prime \prime \prime \prime}+\frac{2}{r} \phi^{\prime \prime \prime}-\frac{\phi^{\prime \prime}}{r^{2}}+\frac{\phi^{\prime}}{r^{3}}=0,
$$

so that a solution $\phi$ to $(3.2)$ must be a linear combination of

$$
1, \quad \ln r, \quad r^{2}, \quad r^{2} \ln r
$$

The linear components of the nominal stress tensor $\mathbf{S}$ that satisfy the Euler equations

$$
\nabla \bullet \mathbf{S}=\mathbf{0}
$$

can then be determined explicitly in terms of $\phi$. Once $\mathbf{S}$ is known the linear displacements $\left(u_{r}^{*}, u_{z}^{*}\right)$ can also be expressed in terms of $\phi$ [see 1, pp. 381-382].

The appropriate $\phi$ must satisfy the following mechanical conservation law. Given a cylinder of arbitrary radius and unit depth centered at the origin, the net vertical force on the lateral surface must equal $\hat{\alpha}$, the magnitude of the vertical line load per unit length. The traction at a point on the lateral surface of the cylinder is given by

$$
\mathbf{S}^{\mathrm{T}} \bullet \mathbf{n}=\mathbf{S}^{\mathrm{T}} \bullet \mathbf{e}_{r}=S_{r r} \mathbf{e}_{r}+S_{z r} \mathbf{e}_{z}
$$


while a differential element of the lateral surface is of the form

$$
d A=r d \theta d z
$$

Thus, the vertical component of force per initial surface area is

$$
S_{z r} r d \theta d z
$$

and the above conservation law can then be represented by the integral relation

$$
\int_{0}^{1} \int_{0}^{2 \pi} S_{z r} r d \theta d z=\hat{\alpha}, \quad \forall r>0 .
$$

Since $S_{z r}$ is independent of $\theta$ and $z$, we have

$$
S_{z r}=\frac{\hat{\alpha}}{2 \pi r}
$$

and the only stress function from (3.3) satisfying (3.9) is

$$
\phi(r)=\frac{\hat{\alpha}}{2 \pi(1-\nu)} \ln r
$$

where $\nu$ is Poisson's ratio. Using (3.10), we find the linear displacements to be

$$
u_{r}^{*}=0
$$

and

$$
u_{z}^{*}=\frac{(1+\nu) \hat{\alpha}}{\pi \mu} \ln r
$$

or in terms of $\bar{\rho}^{*}$ and $\omega^{*}$,

$$
\bar{\rho}^{*}(\rho) \stackrel{L}{=} \rho
$$

and

$$
\omega^{*}(\rho) \stackrel{L}{=} \frac{(1+\nu)}{\pi} \ln \rho
$$

where the superposed $L$ denotes the linear solution.

4. Classes of nonlinearly elastic solutions. Upon computing the variation of the right side of (2.15) and integrating by parts, we obtain an expression of the form

$$
\delta \Pi=\int_{0}^{\infty}\left(\mathrm{E}_{\bar{\rho}} w \delta \bar{\rho}+\mathrm{E}_{\omega} w \delta \omega\right) \rho d \rho-\delta \omega(0) .
$$

By standard arguments, (4.1) yields the following Euler equations:

$$
\begin{aligned}
& \mathrm{E}_{\bar{\rho}} w \equiv \rho \frac{\partial w}{\partial \bar{\rho}}-\frac{d}{d \rho}\left[\rho \frac{\partial w}{\partial \bar{\rho}^{\prime}}\right]=0 \\
& \mathrm{E}_{\omega} w \equiv \frac{d}{d \rho}\left[\rho \frac{\partial w}{\partial \omega^{\prime}}\right]=0
\end{aligned}
$$


This last equation implies

$$
\frac{\partial w}{\partial \omega^{\prime}}=\frac{k}{\rho}
$$

where $k$ is a constant to be determined. Since the variational functional $(2.15)$ is to model an infinite medium, there are no physical boundaries and thus no natural boundary conditions for (4.3) and (4.4).

As remarked in Sec. 1, the linear solution (3.12) or (3.14) predicts an infinite displacement under the load and thus violates the basic premise of linear elasticity. We wish to clarify the nature of this singularity, i.e., determine if it is inherent to the problem or merely an artifact of the linearized theory, by studying the nonlinear version of the problem. In addition, as with the nonlinearly elastic Boussinesq problem treated in [6], we cannot gain insight into the nonlinear line load problem by attempting a linearization. This is due to the fact that there exists a single intrinsic length $\hat{\alpha} / \mu$ so that nondimensionalizing renders the governing equations parameter free. Thus, our purpose is first to determine if there exist classes of compressible nonlinear elastic strain energy density functions for which the linear solution (3.13) and (3.14) is a possible solution to the governing equations for the nonlinearly elastic line load problem. Then we wish to show that among these quite general material models that allow the linear solution is a form which can be used to describe a variety of commonly used strain energies proposed in the literature (see Eq. (4.28)). Substituting the linear radial solution (3.13) into (2.8) results in

$$
I_{3}=1 \text {. }
$$

Equation (4.5) implies that the linear solution to the line load problem can hold only for those compressible materials capable of sustaining volume preserving (isochoric) deformations. The remaining two strain invariants $(2.6)$ and $(2.7)$ reduce to

$$
\alpha \equiv I_{1}=I_{2}=\frac{(1+\nu)^{2}}{\pi \rho^{2}}+3
$$

when evaluated at the linear solution (3.13), (3.14). Consider now the Euler equations (4.2) and (4.4) associated with

$$
w=w\left(I_{1}, I_{2}, I_{3}\right)
$$

the strain-energy density for isotropic elastic compressible materials. On employing the chain rule and substituting (3.13) and (3.14) for $\bar{\rho}$ and $\omega$ respectively, these reduce drastically to

$$
2 \bar{w}_{11}+6 \bar{w}_{12}+2 \bar{w}_{13}+4 \bar{w}_{22}+2 \bar{w}_{2: 3}+\bar{w}_{2}=0
$$

and

$$
2 \bar{w}_{1}+2 \bar{w}_{2}=k .
$$

In (4.8) and (4.9), $\bar{w}_{i j}=\frac{\partial^{2} w}{\partial I_{i} \partial I_{j}}$, where the superposed bar indicates (here and subsequently) that these expressions are evaluated at the values of $I_{1}, I_{2}$, and $I_{33}$ given in (4.5) and (4.6).

Equations (4.8) and (4.9) represent conditions that (4.7) must meet for (3.13) and (3.14) to be a solution to the line load problem. We now demonstrate a procedure that 
uses (4.8) and (4.9) to determine more specific forms of (4.7), and thus special classes of materials that have (3.13) and (3.14) as a solution.

If (4.8) and (4.9) are considered as linear partial differential equations with respect to the $I_{i}$, a possible solution technique is separation of variables. With this as motivation, we first consider a material of the form

$$
w=\frac{\mu}{2} M\left(I_{1}\right) N\left(I_{2}\right) P\left(I_{3}\right)
$$

To guarantee that the strain-energy vanishes in the undeformed state we require either

$$
N(3)=0 \quad \text { or } \quad M(3)=0 \text {. }
$$

Condition (4.9) implies that

$$
\bar{M}_{I_{1}} \bar{N}+\bar{M} \bar{N}{, I_{2}}_{2}=\frac{k}{\mu \bar{P}}
$$

for the strain energy (4.10), where the right-hand side of (4.12) is constant by virtue of (4.5). In general, the invariants $I_{1}$ and $I_{2}$ clearly are not identical (see, e.g., (2.6), (2.7)). However, this is not the case when considering the deformation associated with the linear solution (3.13), (3.14), and the left-hand side of (4.12) is thereby rendered a function of the single variable (4.6). Thus, it is useful to now consider the functions $\bar{M}$ and $\bar{N}$ as functions of the single independent variable $\alpha$ to determine what restrictions (4.12) implies for (4.10). Equation (4.12) thus becomes

$$
\bar{M}^{\prime} \bar{N}+\bar{M} \bar{N}^{\prime}=\frac{\tilde{k}}{\bar{P}}
$$

where $\left(^{\prime}\right)=\frac{d}{d \alpha}$ and $\tilde{k}=\frac{k}{\mu}$. Integration of (4.13) yields

$$
\bar{N}=\left(\frac{\tilde{k}}{\bar{P}} \alpha+c\right) \frac{1}{\bar{M}}
$$

where $c$ is a constant of integration. The condition $\bar{N}(3)=0$ yields $c=-3 k / \bar{P}$. On using (4.12) and (4.14), the condition (4.8) for the material (4.10) becomes

$$
3 \alpha \bar{M} \bar{M}^{\prime \prime}+3 \alpha \bar{M}^{\prime 2}+\left(\frac{\alpha}{2}+7\right) \bar{M} \bar{M}^{\prime}+\frac{3}{\bar{P}^{2}}\left[\frac{2 \bar{M}^{\prime \prime}}{\bar{M}}-\frac{2 \bar{M}^{\prime 2}}{\bar{M}^{2}}-\frac{\bar{M}^{\prime}}{\bar{M}}\right]=\frac{\bar{P}^{\prime}}{\bar{P}^{3}},
$$

where we note that $\bar{P}=P(1)$ and $\bar{P}^{\prime}=P^{\prime}(1)\left(P^{\prime} \equiv d P / d I_{3}\right)$ are constants. Suppose (4.15) is solved for $\bar{M}(\alpha)$ (possibly numerically), and then $\bar{N}(\alpha)$ is determined from (4.14). If $\alpha$ is then replaced by $I_{1}$ and $I_{2}$ in the resulting equations for $\bar{M}$ and $\bar{N}$ respectively, then

$$
w=\frac{\mu}{2} \bar{M}\left(I_{1}\right) \bar{N}\left(I_{2}\right) P\left(I_{3}\right)
$$


determines a class of strain-energy densities that will have (3.13) and (3.14) as a solution, where $P\left(I_{3}\right)$ is arbitrary except that $P(1) \neq 0$.

We next consider materials of the form

$$
w=\frac{\mu}{2}\left[P_{1}\left(I_{3}\right) M\left(I_{1}\right)+P_{2}\left(I_{3}\right) N\left(I_{2}\right)+P_{33}\left(I_{3}\right)\right],
$$

which can be used to represent a number of models proposed in the literature. To guarantee that the strain energy vanishes in the undeformed state we require that

$$
M(3)=N(3)=P_{3}(1)=0 .
$$

Condition (4.9) implies that for materials of the form (4.17),

$$
\bar{P}_{1} \bar{M}_{I_{1}}+\bar{P}_{2} \bar{N}_{I_{2}}=\frac{k}{\mu} .
$$

As before, it is expedient to treat $M$ and $N$ as functions of the single variable $\alpha$ so that Eq. (4.19) may be written as

$$
\bar{P}_{1} \bar{M}^{\prime}+\bar{P}_{2} \bar{N}^{\prime}=\tilde{k}
$$

Integration of (4.20) yields

$$
\bar{N}=-\bar{M} \frac{\bar{P}_{1}}{\bar{P}_{2}}+\frac{\tilde{k}}{\bar{P}_{2}} \alpha+\frac{c}{\bar{P}_{2}}
$$

where $c$ is a constant of integration. Recalling (4.18), if $\bar{M}(3)=0$, then $\bar{N}(3)=0$ requires $c=-3 \tilde{k}$. Using (4.19) and (4.21), condition (4.8) applied to (4.17) may be written as

$$
2 \bar{P}_{1} \bar{M}^{\prime \prime}+\left[\frac{2 \bar{P}_{1} \bar{P}_{2}^{\prime}}{\bar{P}_{2}}-2 \bar{P}_{1}^{\prime}+\bar{P}_{1}\right] \bar{M}^{\prime}=\tilde{k}\left(2+\frac{\bar{P}_{2}^{\prime}}{\bar{P}_{2}}\right) .
$$

Note that $\bar{P}_{1}=P_{1}(1), \bar{P}_{2}=P_{2}(1), \bar{P}_{1}^{\prime}=P_{1}^{\prime}(1)$, and $\bar{P}_{2}^{\prime}=P_{2}^{\prime}(1)$ are constants. Equation (4.22) is a first-order linear ordinary differential equation for $\bar{M}^{\prime}(\alpha)$ which can be integrated yielding

$$
\begin{aligned}
\bar{M}= & k_{2} \exp \left[-\alpha\left(\frac{\bar{P}_{2}^{\prime}}{\bar{P}_{2}}-\frac{\bar{P}_{1}^{\prime}}{\bar{P}_{1}}+\frac{1}{2}\right)\right]+k_{1} \\
& +\frac{\tilde{k}}{2} \frac{2 \alpha \bar{P}_{1} \bar{P}_{2}^{\prime 2}+\left[(5 \alpha-2) \bar{P}_{1}-2 \alpha \bar{P}_{1}^{\prime}\right] \bar{P}_{2} \bar{P}_{2}^{\prime}+\left[(2 \alpha-4) \bar{P}_{1}-4 \alpha \bar{P}_{1}^{\prime}\right] \bar{P}_{2}^{2}}{4 \bar{P}_{1}^{2} \bar{P}_{2}^{\prime 2}+\left(4 \bar{P}_{1}^{2}-8 \bar{P}_{1} \bar{P}_{1}^{\prime}\right) \bar{P}_{2} \bar{P}_{2}^{\prime}+\left(4 \bar{P}_{1}^{\prime 2}-4 \bar{P}_{1} \bar{P}_{1}^{\prime}+\bar{P}_{1}^{2}\right) \bar{P}_{2}^{2}},
\end{aligned}
$$

where $k_{1}$ and $k_{2}$ are constants of integration. To make $M(3)=0$, choose

$$
\begin{aligned}
k_{1}= & k_{2} \exp \left[-3\left(\frac{\bar{P}_{2}^{\prime}}{\bar{P}_{2}}-\frac{\bar{P}_{1}^{\prime}}{\bar{P}_{1}}+\frac{1}{2}\right)\right] \\
& +\frac{\tilde{k}}{2} \frac{6 \bar{P}_{1} \bar{P}_{2}^{\prime 2}+\left[13 \bar{P}_{1}-6 \bar{P}_{1}^{\prime}\right] \bar{P}_{2} \bar{P}_{2}^{\prime}+\left[\left(2 \bar{P}_{1}-12 \bar{P}_{1}^{\prime}\right] \bar{P}_{2}^{2}\right.}{4 \bar{P}_{2}^{\prime 2}+\left(4 \bar{P}_{1}^{2}-8 \bar{P}_{1} \bar{P}_{1}^{\prime}\right) \bar{P}_{2} \bar{P}_{2}^{\prime}+\left(4 \bar{P}_{1}^{\prime 2}-4 \bar{P}_{1} \bar{P}_{1}^{\prime}+\bar{P}_{1}^{2}\right) \bar{P}_{2}^{2}}
\end{aligned}
$$


$N(\alpha)$ is then determined from (4.21) on using (4.23) and (4.24). Substituting (4.23) for $\bar{M}$ in (4.21) and then replacing $\alpha$ by $I_{1}$ and $I_{2}$ in $\bar{M}$ and $\bar{N}$, respectively,

$$
w=\frac{\mu}{2}\left[P_{1}\left(I_{3}\right) \bar{M}\left(I_{1}\right)+P_{2}\left(I_{3}\right) \bar{N}\left(I_{2}\right)+P_{3}\left(I_{3}\right)\right]
$$

determines a class of strain-energy densities that will admit (3.13) and (3.14) as a solution, where the $P_{i}\left(I_{3}\right)$ are arbitrary except that $P_{3}(1)=0$. For example, suppose $P_{i}\left(I_{3}\right)$ $(i=1,2)$ are constants and $k_{2}=0$ in (4.23). Then (4.23) reduces to

$$
\bar{M}(\alpha)=\frac{\tilde{k}(\alpha-3)}{\bar{P}_{1}},
$$

and substitution of (4.26) into (4.21) yields

$$
\bar{N}(\alpha)=0
$$

Using (4.26) and (4.27) it can be shown that a strain-energy density of the form

$$
w=\frac{\mu}{2}\left[c_{1}\left(I_{1}-3\right)+P_{3}\left(I_{3}\right)\right]
$$

will have (3.13) and (3.14) as a solution to the line load problem. A variety of materials proposed in the literature are of the form (4.28) (see [9] for a partial list). We remark that this process could be continued by choosing other forms for the strain-energy density and then obtaining restrictions on these forms.

We conclude by identifying an interesting comparison between the line load problem and the problem involving axisymmetric anti-plane shear of isotropic compressible nonlinearly elastic circular tubes studied in [9]. In [9], a circular tube centered on the $z$-axis and bonded on its inner surface to a rigid cylinder is subjected to a uniform axial shear traction on its outer surface. First, the invariants associated with both the axial shear problem (radial deformation possible) and the anti-plane shear problem (no radial deformation) take on the same form as (2.6)-(2.8) and (4.5)-(4.6), respectively (see Eqs. (2.12)-(2.14) and (3.1) of [9]). Specifically, it is shown in [9] that classes of compressible materials capable of sustaining axisymmetric anti-plane shear must undergo a volume-preserving deformation, as was the case here for the line load problem (see (4.5)). Additionally, the two necessary conditions on the strain energy density function deteimined in [9] for axisymmetric anti-plane shear to be possible reduce directly to (4.8) and (4.9) when the out-of-plane displacement is set equal to the corresponding linear (natural log) solution similar to that given by (3.14). In particular, analogous results for the two problems are obtained for materials of the form (4.28). While this may at first seem surprising, the two problems are closely related in the following way. The problem of a tube subjected to a uniform axial shear traction on its inner surface while the outer surface is held fixed is equivalent to the problem examined in [9]. If the inner radius is allowed to approach zero, and the outer radius approaches infinity, the physical connection between the line load problem and the axial shear problem becomes apparent. Thus, in particular, the technique developed here applied to the line load problem (formally) recovers, in a limiting sense, results obtained in [9] for anti-plane shear deformations of a tube composed of a nonlinearly elastic material described by (4.28). 
Acknowledgments. The authors wish to thank J. Simmonds whose influence sparked our interest in this problem. The authors are also grateful to the reviewer of this paper for the helpful comments and suggestions given.

\section{REFERENCES}

[1] S. P. Timoshenko and J. N. Goodier, Theory of Elasticity, third edition, McGraw-Hill, Inc., New York, 1970

[2] R. W. Little, Elasticity, Prentice-Hall, Inc., 1973

[3] I. S. Sokolnikoff, Mathematical Theory of Elasticity, McGraw-Hill, Inc., New York, 1956

[4] J. L. Sanders, Note on the Mindlin problem, Mechanics of Material Behavior, Elsevier, New York, 1984, pp. 345-349

[5] P. G. Warne, Ph. D. Dissertation, The Nonlinearly Elastic Boussinesq Problem and Lie Groups, University of Virginia, Charlottesville, VA, May 1993

[6] J. G. Simmonds and P. G. Warne, Notes on the nonlinearly elastic Boussinesq problem, Journal of Elasticity 34, 69-82 (1994)

[7] J. G. Simmonds, A necessary condition on the string-energy density for a circular, rubber-like plate to have a finite deflection under a concentrated load, J. Appl. Mech. 56, 484-486 (1989)

[8] J. G. Simmonds and M. A. Horn, Asymptotic analysis of the nonlinear equations for an infinite, rubber-like slab under an equilibrated vertical line load, J. Elasticity 24, 105-127 (1990)

[9] D. A. Polignone and C. O. Horgan, Axisymmetric finite anti-plane shear of compressible nonlinearly elastic circular tubes, Quart. Appl. Math. 50, 323341 (1992)

[10] A. E. Green and W. Zerna, Theoretical Elasticity, second edition, Oxford, 1968

[11] J. M. Ball, Convexity conditions and existence theorems in nonlinear elasticity, Arch. Rational Mech. Anal. 63, 337-403 (1977)

[12] R. W. Ogden, Non-Linear Elastic Deformations, Ellis Horwood Limited, England, 1984 